\title{
A novel Y160C mutation of Keratin 10 gene in a Chinese male infant with epidermolytic hyperkeratosis
}

\author{
Chenyu Zhao ${ }^{1}$, Yonggui $\mathrm{Li}^{1}$, Gaoxing Shi ${ }^{2}$, Xiaoliu Shi ${ }^{1,2}$, Guiying Zhang \\ Departments of ${ }^{1}$ Gastroenterology, ${ }^{2}$ Medical Genetics, and ${ }^{3}$ Dermatology, The Second Xiangya Hospital of Central South \\ University, Changsha, 410011, China. E-mail: zhangguiying67@aliyun.com \\ Received: 16th July 2017, Accepted: 30th August 2017
}

SUMMARY: Zhao C, Li Y, Shi G, Shi X, Zhang G. A novel Y160C mutation of Keratin 10 gene in a Chinese male infant with epidermolytic hyperkeratosis. Turk J Pediatr 2018; 60: 426-428.

Epidermolytic hyperkeratosis (EHK) is a rare genodermatosis whose prevalence is less than 1 in 100,000 . Mutations in either the keratin 1 or keratin 10 genes lead to EHK characterized by congenital erythema and epidermal blisters at birth, followed by hyperkeratotic skin lesions with age. We here report a 1-and-a-half-year-old male infant with EHK caused by a novel mutation, c. $479 \mathrm{~A}>\mathrm{G}$, g.489A $>$ G, p. Y160C, of the keratin 10 gene. Mutation at this position has been reported previously, but the type of amino acid change was different. These results expand the database of keratin 10 gene mutations.

Key words: hyperkeratosis, epidermolytic, genodermatosis, keratin-10, mutation.

Epidermolytic hyperkeratosis (EHK, MIM\#113800), also called bullous congenital ichthyosiform erythroderma (BCIE) or epidermolytic ichthyosis (EI), is a rare autosomal dominant genodermatosis caused by the defects in keratin 1 or keratin 10 , encoded by keratin 1 or 10 genes, which serve as cytoskeletons of epithelial keratinocytes. ${ }^{1}$ Clinical features of EHK whose incidence is less than 1 in 100,000 include congenital erythroderma and blistering, later replaced by progressive hyperkeratosis. ${ }^{2}$ To date, the Human Gene Mutation Database (HGMD; http://www. hgmd.cf.ac.uk/ac/index.php) has collected 120 mutations of keratin 1 and keratin 10 genes. In $\mathrm{EHK}$, the rate of spontaneous mutation can reach $50 \% .^{3}$

\section{Case Report}

A 1-and-a-half-year-old male Chinese infant was born with generalized blistering and erythema. Erythematous hyperkeratotic skin lesions were amongst the major clinical features. Physical examination showed erythema and thickened, brown, desquamated hyperkeratosis on his trunk and extremities (Fig. 1a). His palms and soles were intact. Skin biopsy from his trunk revealed epidermal hyperkeratosis, degeneration in the granular layer, and intracellular edema in the spinous layer (Fig. 1b). He was diagnosed with EHK. His parents, who are as of this writing unaffected, are a non-consanguineous couple (Fig. 2). There was no similar family history in the patient's pedigree. In order to evaluate the clinical diagnosis and to discern the genetic etiology, blood samples were obtained for genetic testing.

We isolated genomic DNA from peripheral blood. Exon-intron boundaries and the exon sequence of the keratin 1 and keratin 10 genes were obtained by direct sequencing. The patient's parents signed written informed consent forms. However, they declined to provide a sample of his sibling's blood. The study was approved by the Ethical Committee. We then detected a heterozygous single nucleotide substitution, c.479A $>$ G, leading to a tryosine to cysteine at codon 160 in exon 1 of the patient's keratin 10 gene in the patient, which was not present in his parents (Fig. 3).

\section{Discussion}

EHK is clinically characterized by neonatal erythroderma and blistering. Later on, generalized scaling hyperkeratosis develop, especially in flexural areas. Generally, EHK 


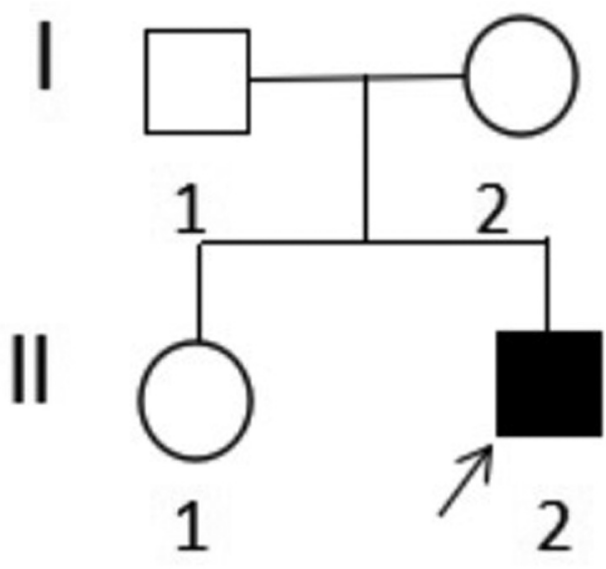

Fig. 1. The EHK pedigree. The arrow denotes the proband.

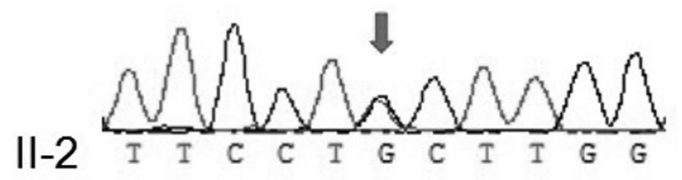

I-1

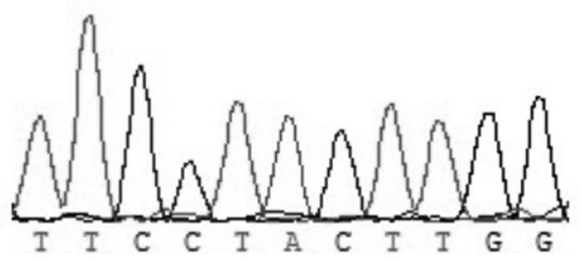

I-2

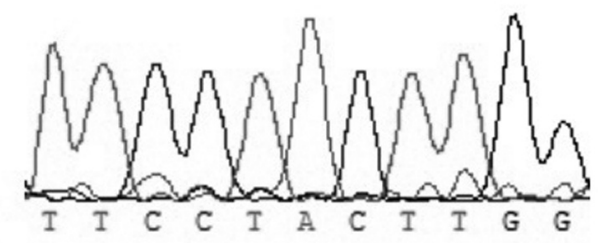

Fig. 2. Sanger sequencing revealed a heterozygous c. 479 $A>G$ mutation (indicated by the arrow) in the proband, while his unaffected parents had wild-type sequences.

is regarded as an autosomal dominant keratinization disorder. But researchers discovered a rare form autosomal recessive $\mathrm{EHK}^{4}$. In addition, there is an uncommon form of EHK termed palmoplantar keratoderma (PPK). PPK that is caused by mutations in keratin 1 , keratin 9 and keratin 16 genes is characterized by hyperkeratosis of palms and soles. Histopathological examination of skin would reveal vacuolar degeneration in the spinous and granular layers of epidermis. Besides, epidermal hyperkeratosis can also be observed under light microscopy. Ultrastructural
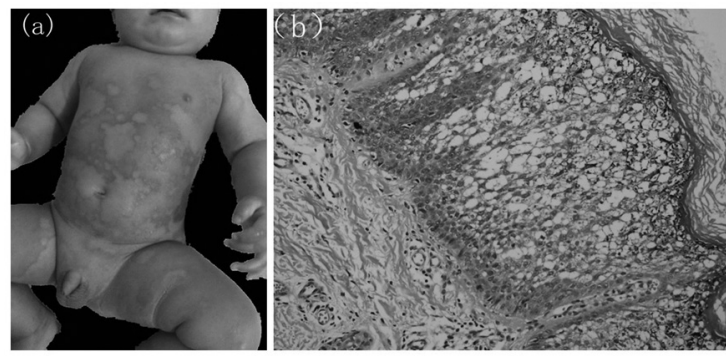

Fig. 3. (a) Clinical manifestations of our patient. There was erythema and hyperkeratosis on his trunk and extremities. (b) Hematoxylin and eosin staining; (original magnification $\times 200$ ) histology features of the patient. Skin biopsy revealed epidermal hyperkeratosis, degeneration in the granular layer, and intracellular edema in the spinous layer

analysis showed aggregating and clumping of the keratin filament bundles. At present, there is no cure for EHK. However, retinoids could reduce hyperkeratosis. ${ }^{5}$

Our patient had typical clinical manifestations and definite pathological diagnosis. Genetic testing demonstrated a disease-causing variant in keratin 10 gene. The variant was not found in either the 1000 Genomes Project (1000G; http:// browser.1000genomes.org/) or in the Exome Aggregation Consortium (EXAC; http://exac. broadinstitute.org/). It is a de novo mutation confirmed in both parents. Arin et al. ${ }^{6}$ reported a patient with a Y160S mutation in the keratin 10 gene with severe clinical features of EHK. We found a different amino acid substitution at this position. Prediction software, specifically MutationTaster ${ }^{7}$, PolyPhen-2 ${ }^{8}$, and SIFT ${ }^{9}$, predicted a deleterious effect of the mutation. Therefore, according to the American College of Medical Genetics and Genomics' standards and guidelines, we classified the variant as a likely pathogenic mutation. ${ }^{10}$ By scanning HGMD and previous published works, we concluded that the variant is a novel mutation.

In conclusion, we described an individual with EHK harboring a novel and likely pathogenic mutation,c.479A $>$ G, g.489A $>$ G, p. Y160C. Our results expanded the database of keratin 10 gene mutations. However, further studies to confirm relations between the variation and EHK are needed.

\section{Acknowledgement}

We would like to thank the members of the patient's family for their participation. 


\section{REFERENCES}

1. Arin MJ. The molecular basis of human keratin disorders. Hum Genet 2009; 125: 355-373.

2. Haruna K, Suga Y, Mizuno Y, et al. R156C mutation of keratin 10 causes mild form of epidermolytic hyperkeratosis. J Dermatol 2007; 34: 545-548.

3. Arin MJ, Oji V, Emmert S, et al. Expanding the keratin mutation database: Novel and recurrent mutations and genotype-phenotype correlations in 28 patients with epidermolytic ichthyosis. Br J Dermatol 2011; 164 : 442-447.

4. Covaciu C, Castori M, De Luca N, et al. Lethal autosomal recessive epidermolytic ichthyosis due to a novel donor splice-site mutation in KRT10. Br J Dermatol 2010; 162: 1384-1387.

5. Lacz NL, Schwartz RA, Kihiczak G. Epidermolytic hyperkeratosis: A keratin 1 or 10 mutational event. Int J Dermatol 2005; 44: 1-6.
6. Arin MJ, Longley MA, Anton-Lamprecht I, et al. A novel substitution in keratin 10 in epidermolytic hyperkeratosis. J Invest Dermatol 1999; 112: 506-508.

7. Schwarz JM, Rodelsperger C, Schuelke M, Seelow D. MutationTaster evaluates disease-causing potential of sequence alterations. Nat Methods 2010; 7: 575-576.

8. Adzhubei IA, Schmidt S, Peshkin L, et al. A method and server for predicting damaging missense mutations. Nat Methods 2010; 7: 248-249.

9. Kumar P, Henikoff S, Ng PC. Predicting the effects of coding non-synonymous variants on protein function using the SIFT algorithm. Nat Protoc 2009; 4: 10731081.

10. Richards S, Aziz N, Bale S, et al; ACMG Laboratory Quality Assurance Committee. Standards and guidelines for the interpretation of sequence variants: A joint consensus recommendation of the American College of Medical Genetics and Genomics and the Association for Molecular Pathology. Genet Med 2015; 17: 405-424. 